\title{
GRAMS: Grid Resource Analysis and Monitoring System ${ }^{1}$
}

\author{
Hongning Dai, Minglu Li, Linpeng Huang, Yi Wang, and Feng Hong \\ Department of Computer Science \& Engineering, Shanghai Jiao Tong University, \\ 1954 Hua Shan Road, 200030 Shanghai, P.R. China \\ \{hndai, li-ml, huang-lp, wangsuper, hongfeng\}@cs.sjtu.edu.cn
}

\begin{abstract}
In this paper we propose GRAMS, a resource monitoring and analysis system in Grid environment. GRAMS provides an infrastructure for conducting online monitoring and performance analysis of a variety of Grid resources including computational and network devices. Based on analysis on real-time event data as well as historical performance data, steering strategies are given for users or resource scheduler to control the resources. Besides, GRAMS also provides a set of management tools and services portals for user not only to access performance data but also to handle these resources.
\end{abstract}

\section{Introduction}

Grids offer us a new vision, infrastructure and trend for the coordinated resources sharing, problem solving and services orchestration in dynamic, multi-institutional virtual organizations [1]. A recent trend in government and academic research is the development and deployment of computational grids [2]. Computational grids are large-scale distributed systems that typically consist of high-performance compute, storage, and networking resources. Several computational grids in China are NHPCE (1999-2001) [7], CNGrid (2002-2006) [8], ChinaGrid (2002-2005) [9], E-Science Grid (2002-2005) [10], Spatial Information Grid (2001-2005) [11] and Shanghai-Grid (a city grid project to enhance the digitalizing of Shanghai)[3].

The primary goal of Shanghai-Grid is to develop a set of system software for the information grid and establish an infrastructure for the grid-based applications. This project will build an information grid tailored for the characteristics of Shanghai and support the typical application of grid-based traffic jam control and guidance. Currently we are trying to build up a system to monitor and control the resources, services and applications that make up the Grid. We have found it difficult to ensure that the resources in the Grid are working correctly to support grid application. It is also cumbersome to predict system performance and to control the resources in the Grid. These difficulties have led to our development of a system for monitoring and analysis of grid resources.

\footnotetext{
${ }^{1}$ Supported by the National Grand Fundamental Research 973 Program of China under Grant No.2002CB312002, the Grand Project (No.03dz15027) and Key Project (No.025115033) of the Science and Technology Commission of Shanghai Municipality.
} 
In this paper, we present a system namely GRAMS (Grid Resource Analysis and Monitoring System) which can observe performance events on each grid node, collect these event data, and analyze performance data to determine what actions should be taken.

\section{GRAMS Architecture}

GRAMS is based on OGSA [5] and GMA [4]. The GRAMS architecture is shown in Fig.1. The GRAMS includes several main components: Monitor Managers, Directory Service, Consumers (Customer Service, Archival Service, and Performance Analysis Service), Steering Service and User Interface. All the services can be deployed to different distributed environments.

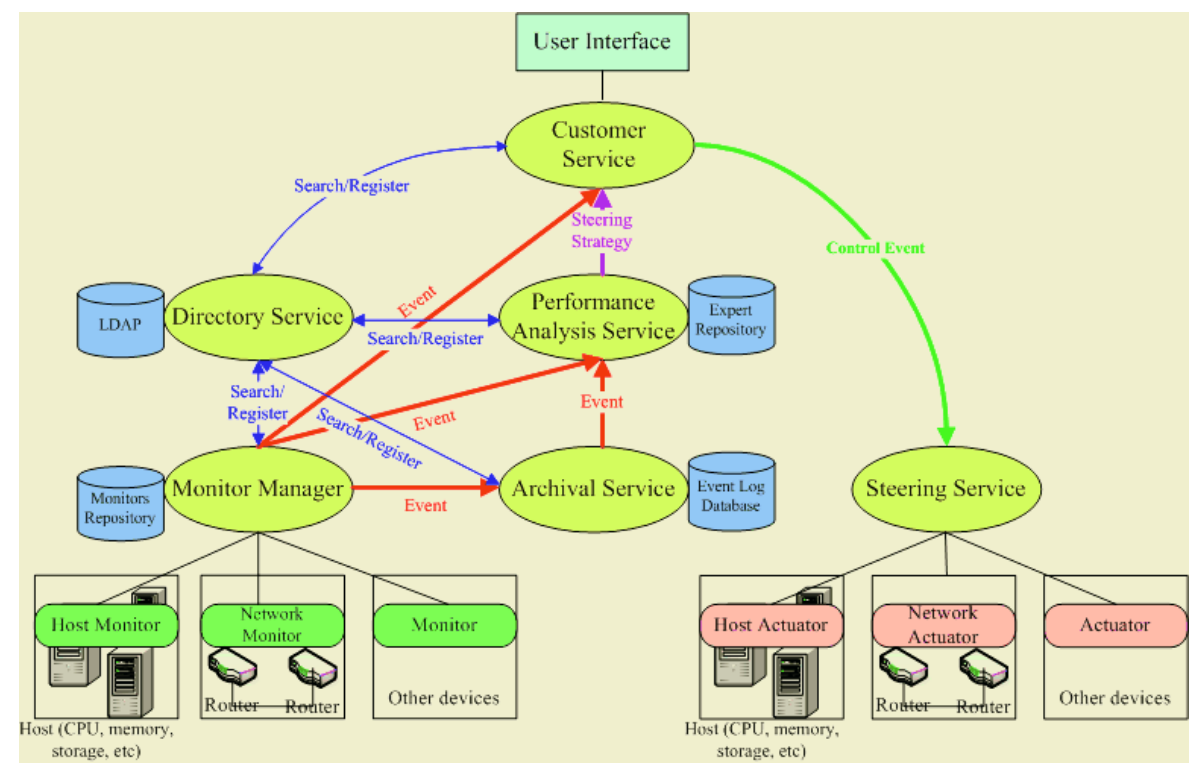

Fig. 1. Architecture of GRAMS framework

Directory Service stores information about producers, consumers and performance data. With the directory service, producers and consumers can publish and discover information that they request. This published information includes registration of producers and consumers, the types of event data, the characteristics of the data, and the ways to gain access to that data. The directory service contains only information about which event instances can be provided or accepted, not the event data itself.

Monitor Managers are used to manage monitors that measure and gather a variety of kinds of information for monitoring and analyzing. The monitors include: host monitor that is used to measure system property, network monitor to catch network info, and other monitor to capture other useful data from other devices of the Grid 
systems. The Monitor Manager publishes the monitors' existences in directory service entries, which tell consumers how to locate them. When the subscription between the monitors and customer service is established, the monitors will send events to customer service through the data channel of Monitor Manager until the subscription is terminated. Monitor repository in Monitor Manager will store the information about monitors (including their locations, their structures and kinds of performance metrics).

Archival Service is a database system which is used to store detailed monitoring information as well as performance results collected. It gained large amounts of longterm event data from Monitor Manager service for later use.

Performance Analysis Service is an expert system which acquires huge amounts of data from Monitor Manager as well as Archival Service, and produces resource management rules and scheduling strategies.

By using User interface provided by Customer Service, users can conduct online monitoring and performance analysis as well as making resource management decisions.

Steering Service receives requests from Customer Service to perform actions on the given resources or devices.

Interactions among GRAMS components and services are divided into controlling streams and data streams. Controlling streams are used to perform tasks including conducting activities of monitors and services, subscribing and querying requests for performance data, registering, querying and receiving information of Directory Service. In data streams delivery, a stream channel is used to transfer data (monitor data, performance data and results) between producers and consumers. Each monitor manager has only one connection to a consumer for delivering all kinds of data. All the connections are secure sockets based on Grid Security Infrastructure (GSI) [6] and Public Key Infrastructure (PKI).

\section{Conclusions}

In this paper we proposed the architecture of GRAMS which is a flexible, scalable monitoring and performance analysis system in Grid environments. We have implemented an early prototype of GRAMS, which already proves it feasible and effect.

There are still a lot works that should be done to improve the system. Fuzzy rules in expert system will be extended to cater for more complex and richer semantic knowledge. Performance prediction and instrumental tracing for Grid-based applications are the centers that our next step work will focus on. We will try to integrate current monitoring tools into GRAMS to serve more types of Grid monitor users and provide more functionalities and features, such as sampling, tracing and profiling [12].

\section{References}

1. Foster, C. Kesselman, and S, Tuecke, "The Anatomy of the Grid: Enable Scalable Virtual Organizations", Int. J. of H. Performance Computing Applications, 15 (2001) pp.200-222.

2. I. Foster and C. Kesselman, The Grid: Blueprint for a New Computing Infrastructure, Morgan Kauffmann, 1999. 
3. M. Li, H. Liu, C. Jiang, and W. Tong, "Shanghai-Grid in Action: The First Stage Projects towards Digital City and City Grid”, LECT NOTES COMPUT SC 3032: 2004, pp.616-623.

4. B. Tierney, R. Aydt, and W. Smith, "A Grid Monitoring Architecture", Technical report, Performance Working Group, Grid Forum, January 2002. http://www-didc.lbl.gov/GGFPERF/GMA-WG/papers/GWD-GP-16-3.pdf

5. I. Foster, C. Kesselman, J. Nick, and S. Tuecke, "Grid Services for Distributed System Integration", IEEE Computer, June 2002, pp.37-46.

6. I. Foster, C. Kesselman, G. Tsudik, and S. Tuecke, "A security architecture for computational grids", In Proceedings of the 5th ACM Conference on Computer and Communications Security (CCS-98), ACM Press, New York, Nov. 3-5 1998, pp.83-92.

7. National High Performance Computing Environ-ment (NHPCE): http://vega.ict.ac.cn

8. China National Grid Project (CNGrid): http://www.grid.org.cn/

9. ChinaGrid (China Education and Research Grid): http://grid.hust.edu.cn/platform/chinagrid.jsp

10. China E-science Grid Project: http://www.most.gov.cn/English/index.htm

11. Spatial Information Grid (SIG):

http://www.nudt.edu.cn/newweb/intercommunion/whatissig.htm

12. Michael Gerndt, et al. "Performance Tools for the Grid: State of the Art and Future", APART-2 White Paper on Grid Performance Analysis, http://www.lpds.sztaki.hu/ zsnemeth/apart/repository/gridtools.pdf. 